\title{
A Network Coding based DTN Convergence Layer Reliable Transport Mechanism over InterPlaNetary Networks
}

\author{
S. Haoliang, L. Lixiang, H. Xiaohui
}

\author{
Sun Haoliang \\ 1. Graduate University of the Chinese Academy of Sciences \\ 19A Yuquanlu,Beijing, 100049, P. R. China \\ 2. National Key Laboratory of Integrated Information System Technology, \\ Institute of Software, Chinese Academy of Sciences \\ 4\# South Fourth Street, Zhong Guan Cun, Beijing 100190 P.R. China \\ E-mail: haoliang08@iscas.ac.cn

\section{Liu Lixiang, Hu Xiaohui} \\ Institute of Software, Chinese Academy of Sciences \\ 4\# South Fourth Street, Zhong Guan Cun Street, Beijing, China, 100190
}

\begin{abstract}
The realization of deep space scientific missions are enabled by the developments in the space technologies. TCP can not provide effective communication service in deep space links because of the long propagation delay and high BERs characteristics.Scientific community propose Delay Tolerant Network (DTN) for resolving the communication problem between earth and other planet. DTN introduces a new layer in the protocol stack called Bundle Layer that is placed between application layer and transport layer.DTN calls the transport protocols that it uses to move data across different networks convergence layers. This study present a novel Network Coding based convergence layer Reliable TransPort mechanism(NC-RTP) in order to provide effective communication service between DTN peers. This mechanism transmit a coded bundle every $\mathrm{M}$ original bundles $(\mathrm{M}$ is related to the packet error rate of the communication link). The coded bundle is a random linear combination of previous $\mathrm{M}$ original bundles. Using coded bundle and (M-1)original bundles, the receiver could decode and generate a single lost bundle of previous $\mathrm{M}$ original bundles. In this way, NC-RTP could compensate any single lost bundle in $(\mathrm{M}+1)$ transmitted bundles(including $\mathrm{M}$ original bundles and 1 coded bundle).Our theoretical and simulation performance evaluation results reveal that NC-RTP can enhance transmit reliability and make file transfered faster.

Keywords: DTN,Convergence Layer, Reliable Transport, Network Coding.
\end{abstract}

\section{Introduction}

The realization of deep space scientific missions are enabled by the developments in the space technologies. The future space exploration includes missions to deep space that require communication among planets, moons, satellites, asteroids, robotic spacecrafts, and crewed vehicles. Significant amount of scientific data to be delivered to the Earth are produced by these missions. The next step in the design and development of deep space networks is expected to be the Internet of the deep space planetary networks ,called InterPlaNetary (IPN) Internet [1].IPN is an architecture envisioned for interconnecting earth with other planets. The purpose of IPN is to build a communication infrastructure between planets and satellites .

InterPlaNetary (IPN) Internet, in contrast to conventional Internet links, are characterized by: 
1. High Link Bit Error Rates. Deep space links have extremely high bit error rates, which may be up to 10 [2], [3].

2. Very High Propagation Delays. The propagation delay increases with the distance. The propagation delay between Earth and Mars varies between 8 and 40 minutes depending on the orbital location of the planets [2] [3].and the propagation delays to outer space planets become even higher.

3. Bandwidth Asymmetry. Asymmetry between the forward and the return path bandwidth may be up to 1000:1 [3] [4].

4. Intermittent Connectivity. Planetary bodies, asteroids or spacecraft may periodically interrupt the communication link between the path endpoints [3] [5].

In this network,classical transport layer mechanisms are not suitable. Scientific community propose Delay Tolerant Network (DTN) [6] for resolving the communication problem between earth and other planet such as Mars. DTN is an architecture particularly useful in scenarios with very long transmission delay or intermittent connectivity, like IPN. DTN introduces a new layer in the protocol stack called Bundle Layer that is placed between application layer and transport layer [7].

The bundle layer resolves the high error rates, long delay, asymmetric data rates and intermittent connectivity by using a store and forward mechanism. It sends a bundle of message fragments to the next-hop node with per-hop error control, which increases the probability of data transmission.

DTN calls the transport protocols that it uses to move data across different networks convergence layers. A convergence layer should make best use of the intermittent and temporary links in DTN. It transfers as much data as possible while the link between peering DTN nodes is up and available. It is widely accepted that regular TCP cannot operate efficiently as a convergence layer transport protocol for InterPlaNetary networks based on DTN architecture [8]. TCP needs 240 minutes to reach Slow Start Threshold equal to 20 packets [5] over a 40 minute Round Trip Time (RTT) path,and longer RTT paths degrade TCP's performance further. Since TCP was designed to operate over wired links, where the link error rate is insignificant, the protocol cannot cope with high link bit error rates [9]. Finally, TCP's transmission rate depends largely on the receiver's feedback.TCP sends one acknowledgment (ACK) for each successfully received data packet. On the presence of bandwidth asymmetry, the large number of ACKs will cause congestion on the reverse path,reducing TCP's transmission rate. As a result, in InterPlaNetary networks based on DTN architecture, a convergence layer transport protocol other than TCP is needed.

The remainder of the paper is organized as follows. Section 2 introduces current convergence layer protocols in DTN architecture and discusses the drawbacks of them. Section 3 proposes our new Network coding based convergence layer reliable transport mechanism(NC-RTP). The theoretical evaluation of NC-RTP is presented in Section 4. And the experimental evaluation is in Section 5. We conclude the paper in Section 6.

\section{Related Works}

There exist already a number of convergence layer transport protocols for InterPlaNetary networks based on DTN architecture. In this section, we briefly review these proposals.

Saratoga [10]is a reliable rate-based UDP/IP file transfer protocol.It is capable of transferring efficiently both small and very large files. It has been developed by Surrey Satellite Technology Ltd (SSTL) used for mission imaging data. Saratoga was designed for dedicated point-to-point links between DTN Peers, which focuses on transferring data efficiently to the next hop when link connectivity is available. Saratoga achieves efficient transmission by sending out data packets 
at the line rate. It also uses a negative acknowledgment strategy in order to deal with channel bandwidth asymmetries. Saratoga is used as a convergence layer to exchange Delay Tolerant Networking bundles [6], [7] between peer nodes [10].

Similarly to Saratoga, the Licklider Transmission Protocol (LTP) [11] is a point-to-point protocol applied as a DTN convergence layer. LTP transfer unnamed blocks of data and introduces the concept of partial reliability by dividing each block of data into two parts: the reliable red part and the unreliable green part. Moreover, LTP send laconic acknowledgments only upon encountering explicit solicitations for reception reports (checkpoints) in the sequence of incoming data segments of the red part of the block. When the communication link is not available, Deferred Transmission is possible as well.

Deep Space Transport Protocol (DS-TP) [12]also can be adapted to serve as an efficient convergencelayer for DTN, by transferring DTN bundles as well as files. It implements a retransmission technique, called Double Automatic Retransmission (DAR), which allows for fast and efficient holefilling at the receiver's buffer. DAR sends each bundle twice,importing some delay between the original transmission and the retransmission. More precisely,one redundant bundle is transmitted every $1 / \mathrm{e}-1$ original bundles(e presents the packet error rate PER).Therefore, in the presence of link errors, corrupted bundles will eventually be replaced by the same correct bundles that arrive later.

Saratoga and LTP achieve efficient transmission by sending out data bundles at the line rate. This ensures that as much data as possible is transferred to the peering node. DS-TP implements Double Automatic Retransmission(DAR) to fast and efficiently compensate lost bundles.

But ,according to the algorithm described in [12], DAR only retransmit finite bundles $((\mathrm{N} /(1 / e-$ 1) bundles , $N$ presents the number of bundles of the file). DAR can not provide effective retransmission for all bundles, a more effective retransmission technique is needed to enhance transmit reliability.

\section{Network coding based convergence layer reliable transport mech- anism}

Network coding is an important approach to enhance transmit reliability in lossy link. The central idea of network coding is to transmit a combination of multi-packet to replace a single packet. The receiver could decode and generate needed packets using received packets. It has a good ability to deal with transmit error [13]. The simplest coding approach is random linear coding. We treat packets as vectors over a finite field. To get a coded packet, random linear coding generates a group of random coefficients vector and perform matrix multiplication with the packets vector. The transmission of coded packets are independent of each other. Generally, to simplify computer programming and memory accessing, the size of finite field is set to 8 , which is the size of a byte. For example, we have two 16 bits data packets to encode, $3 \mathrm{~A} 47 \mathrm{H}$ and $5 \mathrm{~F} 33 \mathrm{H}($ hex), each packet is considered to be a vector with size 2 . Choose two decimal coefficients randomly,4, 30(the coefficients range from 0 to 255). The coded packet is $43 \mathrm{~A} 47 \mathrm{H}+305 \mathrm{~F} 33 \mathrm{H}=\mathrm{E} 91 \mathrm{CH}+27 \mathrm{FAH}=1116 \mathrm{H}$.

Using network coding in DTN convergence layer can enhance transmit reliability and transmit as many bundles as possible before get any ACK information from the receiver. In our study, we transmit a coded bundle every $1 / e-1$ original bundles(e presents the packet error rate PER). The coded bundle is a random linear combination of previous $1 / e-1$ original bundles.Considering original bundles are liner independent with each other,the coefficients can be set to 1 to all original bundles in the coded bundle. Theoretically, every $1 / e$ bundles will lost one bundle under PER e. Using $1 / e-1$ original bundles and the coded bundle of $1 / e-1$ original bundles, we can decode 
and generate the lost original bundle with Gaussian elimination. Thus we can say ,in this way ,the receiver could get all $1 / e-1$ original bundles using received $1 / e-1$ bundles(including original and coded bundles). This approach is called Network Coding based convergence layer Reliable TransPort mechanism(NC-RTP). This mechanism could compensate lost bundle forwardly.

We use an example to illustrate NC-RTP. Let $e=0.2$, so $1 / e-1=4$. A coded bundle is generated and send every 4 bundles.Let $b_{n}$ presents the $n_{t h}$ bundle.The transmission sequence is $b_{1}, b_{2}, b_{3}, b_{4}, b_{1,2,3,4}\left(b_{1,2,3,4}\right.$ presents the coded bundle of $b_{1}, b_{2}, b_{3}, b_{4}$ and $\left.b_{1,2,3,4}=b_{1}+b_{2}+b_{3}+b_{4}\right)$. Theoretically, 1 bundle will be lost during the transmission. Suppose $b_{3}$ is lost, and the reciver get $b_{1}, b_{2}, b_{4}$, and $b_{1,2,3,4}$. Since $b_{1,2,3,4}=b_{1}+b_{2}+b_{3}+b_{4}$ and we have $b_{1}, b_{2}, b_{4}, b_{3}=b_{1,2,3,4}-$ $b_{1}-b_{2}-b_{4}$,in this way we can decode and generate b3 .

Compare to DS-TP, NC-RTP could compensate any single lost bundle in $1 / e-1$ original bundles, and transmit coded bundles ( $N$ presents the number of bundles of the file ) could make all bundles in the file have the chance to be compensated. In this way, NC-RTP can reduce the number of lost bundles during the transport procedure and enhance the transport reliability. Moreover, we generate coded bundle using bundles, the coding and decoding delay is neglectable comparing to the propagation delay of interplanetary links. Because of the long propagation delay of interplanetary links, using NC-RTP can enhance the transmit reliability and complete file transfers faster.

In the next part of this section, we describe in detail the operation process of Network Coding based convergence layer Reliable TransPort mechanism (NC-RTP),followed by pseudo-code of the algorithm.

The initial value of PER e can be set by previous transmit experience. In the transmit process, the sender can compute the value of e depending on the acknowledgement information from the receiver and change it. In this study, we consider the PER is fixed during the transmit process to simplify the analysis.

Sender procedure:The sender transmit bundles in order and transmit a redundant coded bundle every $1 / e-1$ original bundles. The coded bundle is a liner combination of previous $1 / e-1$ original bundles, all coefficients are set to 1 . When the sender get SNACK, retransmit lost bundle according to the acknowledgement information. In order to compensate lost bundle, use NC-RT in the retransmit operation, send a redundant coded bundle every $1 / e-1$ original bundles.

Receiver procedure:When the receiver get bundles, it put original and coded bundles into its receiving queue. When it get accumulative $A C K_{-} D E L A Y$ ( $A C K_{-} D E L A Y$ is set to the ratio of forward and reverse bind with of the link. When the file to be transmitted has few bundles,we can reduce $A C K \_D E L A Y$ to provoke send SNACK procedure)bundles ,send SNACK procedure is provoked.

Send SNACK procedure:When send SNACK procedure is provoked, the sender check original and coded bundles in its receiving queue, decode and generate lost bundle using original and coded bundles, put generated bundle into receiving queue. Check the receiving queue again, if there is any lost bundle,write the lost bundle information into the SNACK bundle and send the SNACK bundle.

The sender side algorithm has to respond to two types of events:the arrival of a bundle from the upper layer, and get SNACK bundle from the receiver. The receiver side algorithm has to respond to the arrival of a bundle.

Source side:

1)Set $S N D \_C O U N T$ to 0.

2)Wait state:If any following events occurs, respond as follows;else, wait.

3)Bundle arrives from upper layer: 
a)Send the bundle to the receiver.

SND_COUNT++.

b) if $\left(\bar{S} N D \_C O U N T==1 / e-1\right)$

Generate a coded bundle with previous1/e - 1bundles.

Send the coded bundle.

$S N D \_C O U N T=0$.

4) Get a SNACK from the receiver:

a) Read the SNACK information,find out which bundle is lost.

b)retransmit the lost bundle,go to 3 ).

Receiver side:

1)Set $R C V$ COUNT to 0 .

2)Set $A C K_{-} D E L A Y$ properly (according to the ratio of the forward and reverse bandwith and the file size).

3)Wait state:If get a bundle from lower layer,respond;else,wait.

4)Bundle arrives from lower layer:

a) $R C V \_C O U T++$;

Add the bundle into the receiving queue,according to the bundle's ID.

b) If $\left(R C V \_C O U N T==A C K \_D E L A Y\right)$

Using the original bundles and the coded bundle to encode the lost bundles.

Enqueue the generated bundle in to the receiving queue.

After the encode operation, if there is any lost bundle,write the

lost bundle information into the SNACK bundle and send the SNACK bundle.

\section{Theoretical Evaluation}

We attempt to evaluate, theoretically, the performance of NC-RTP.In order to derive some initial evaluation results regarding the performance of the NC-RTP, we assume that the link error rate remains constant during the file transfer.

In particular, we consider the Fixed-Rate Transport Protocol (FRTP), whose main functionality is summarized as follows: the FRTP sender sends data on a fixed rate according to the pre-scheduled line rate. The FRTP receiver,responds with SNACKs in order to signal for lost bundle in the receiving queue.

To simplify the analysis,we consider that SNACKs are sent back to the sender without lost,all acknowledgement information are sent back to the receiver side successfully. We evaluate the performance of the aforementioned protocols over a simple one-hop topology. Such topology represents a point-to-point links between DTN Peers in interplanetary networks.Obviously, the primary metric of interest is the time required for the whole file to be delivered at the receiver side.

We define a Round to be the end-to-end transmission of a specific amount of data. A file transfer consists of several Rounds, during the first Round the original file is transmitted, while during the rest of the Rounds, the sender retransmits bundles lost in previous Rounds.

Assume the file consists of $N$ bundles. The link PER is $e$. The FRTP sender will begin the transmission of the file at the channel rate. After completion of the first round the sender will have transmitted $\mathrm{N}$ bundles. During the first round, $N \cdot e$ bundles are lost and will need to be retransmitted during the second round. Similarly, $N \cdot e^{2}$ bundles are lost during the second round and need to be retransmitted during the third round. During the $n_{t h}$ round, the FR-TP sender will need to retransmit $N \cdot e^{n}$ bundles. We assume that when $N \cdot e^{n}<1$ the file transfer is complete. 
Therefore, FRTP needs $n_{F R T P}$ rounds in order to complete the file transfer:

$$
n_{F R T P}=\log _{e}^{e^{n}}=\log _{e}^{\frac{1}{N}}=\frac{\ln \frac{1}{N}}{\ln e}
$$

Besides FRTP,we also consider DSTP to evaluate NC-RTP's performance. According to its operational properties, DSTP will transmit both original and redundant bundles at the line rate. More precisely,one redundant bundle is transmitted every $1 / e-1$ original bundles. Let $r_{1}$ be the number of transmitted redundant bundles during first round, $r_{1}=N /(1 / e-1)$. It is to say, there are $r_{1}$ bundles that were sent twice, and $N-r_{1}$ bundles that were send only once. We assume that the number of bundles lost during the first round (and need to be retransmitted during the second round) equals $a_{1}$ where:

$$
a_{1}=\left(N-r_{1}\right) \cdot e+r_{1} \cdot e^{2}
$$

Substituting $r_{1}$ into Equation 2, we get that:

$$
a_{1}=N \cdot e \cdot(1-e)
$$

Assume that the number of bundles lost during the second round equals $a_{2}$ where:

$$
a_{2}=N \cdot e^{2} \cdot(1-e)^{2}
$$

Generalizing Equations 3 and 4, we assume that during the $n_{t h}$ round, the DS-TP sender will need to retransmit $a_{n}$ bundles, where

$$
a_{n}=N \cdot e^{n} \cdot(1-e)^{n}
$$

The file transfer is complete, once the following equation holds:

$$
\begin{gathered}
N \cdot e^{n} \cdot(1-e)^{n}<1 \\
n_{D S T P}=\log _{e \cdot(1-e)}^{(e \cdot(1-e))^{n}}=\log _{e \cdot(1-e)}^{\frac{1}{N}}=\frac{\ln \frac{1}{N}}{\ln (e \cdot(1-e))}
\end{gathered}
$$

We add NC-RTP on FRTP, called FRTP-NC. FRTP-NC transmits redundant coded bundles every $1 / e-1$ original bundles. The coded bundle is a liner combination of previous $1 / e-1$ original bundles.According to its operational properties, FRTP-NC will transmit both original and coded bundles at the line rate. In ideal condition, every $1 / e-$ bundles (including $1 / e-1$ original bundles and one coded bundles) will lost only one bundle. In this way, the redundant coded bundle will compensate the lost bundle, the file transfer wiil complete in just one round. However, when more than one bundles are lost in every $1 / e$ bundles, the redundant coded bundle can not compensate all the lost bundles, the sender needs to retransmit lost bundles. In this case, the coded bundle can replace one lost bundle, and the sender needs to retransmit less bundles.

Let $a=1 / e$,we divide the whole file into $N /(a-1)$ parts. All parts are independent of each other. In a single part, if only one bundle is lost(including original and coded bundles), no retransmission is needed. If $k(k \leqslant a)$ bundles are lost, only $k-1$ bundles are needed to retransmit.

Let $p_{k}$ presents the probability of $k$ bundles retransmission in a single part(lost $k+1$ bundles).

$$
p_{k}=C_{a}^{k+1} \cdot e^{k+1} \cdot(1-e)^{a-k-1}
$$

The expectation of $k$ presents the number of bundles needs to retransmit in a single part.

$$
E(k)=\sum_{k=1}^{a-1} k \cdot p_{k}=\sum_{k=1}^{a-1} k \cdot C_{a}^{k+1} \cdot e^{k+1} \cdot(1-e)^{a-k-1}
$$


Let $k+1=j$, we get

$$
E(k)=\sum_{j=2}^{a}(j-1) \cdot C_{a}^{j} \cdot e^{j} \cdot(1-e)^{a-j}=\sum_{j=2}^{a} j \cdot C_{a}^{j} \cdot e^{j} \cdot(1-e)^{a-j}-\sum_{j=2}^{a} C_{a}^{j} \cdot e^{j} \cdot(1-e)^{a-j}
$$

Because $\sum_{j=0}^{a} j \cdot C_{a}^{j} \cdot e^{j} \cdot(1-e)^{a-j}=a \cdot e=1, \sum_{j=0}^{a} C_{a}^{j} \cdot e^{j} \cdot(1-e)^{a-j}=(e+1-e)^{a}=1$, and $a=1 / e$,

$$
E(k)=1-(1-e)^{1 / e-1}-\left[1-(1-e)^{1 / e}-(1-e)^{1 / e-1}\right]=(1-e)^{1 / e}
$$

Consider the whole file consists of $N /(a-1)$ parts, in the first transmission, $E(k) \cdot N /(a-1)$ bundles need to retransmit. Let $y_{1}$ be the number of transmitted redundant bundles during first round.

$$
y_{1}=E(k) \cdot N /(a-1)=(1-e)^{1 / e} \cdot N /(1 / e-1)=N \cdot e \cdot(1-e)^{1 / e-1}
$$

Generalizing Equation 12, we assume that during the $n_{\text {th }}$ round, the FRTP-NC sender will need to retransmit $y_{n}$ bundles, where

$$
y_{n}=N \cdot e^{n} \cdot(1-e)^{n \cdot(1 / e-1)}
$$

The file transfer is complete, once the following equation holds:

$$
\begin{gathered}
y_{n}=N \cdot e^{n} \cdot(1-e)^{n \cdot(1 / e-1)}<1 \\
n_{F R T P-N C}=\log _{e \cdot(1-e)^{(1 / e-1)}}^{e^{n} \cdot(1-e)^{n \cdot(1 / e-1)}}=\frac{\ln \frac{1}{N}}{\ln e \cdot(1-e)^{(1 / e-1)}}
\end{gathered}
$$

We use two figures to evaluate the performance of FRTP, DSTP and FRTP-NC. Figure 1 presents the numbers of rounds of FRTP, DSTP and FRTP-NC in different PERs $(N=$ 100000). Figure 2 presents the numbers of rounds of FRTP, DSTP and FRTP-NC in different file sizes $(P E R=0.25)$.

From Figure 1 we observe that, when file size is fixed, for small error rates , the three protocols perform similarly. As the link error rate increases, the rounds that FRTP needs grows sharply, and the rounds that FRTP-NC and DSTP need grow slowly, and FRTP-NC always needs less rounds than DSTP. Only when PER $=0.5$,DSTP and FRTP-NC have the same performance. At this time ,DSTP and FRTP-NC operate in the same way(transmit each bundle twice).

From Figure 2 we observe that, when the link error rate is fixed, the growth of the file size results in the increment of the rounds that three protocols need, but FRTP-NC is the lowest.

Our theoretical evaluation revealed that, FRTP-NC that using NC-RTP needs less time to complete file transmission and it have a better ability to handle high PER and large files in interplanetary networks. NC-RTP can be used in convergence layer to achieve efficient transmission between peer nodes .

\section{Experimental Evaluation}

In this section, we evaluate the performance of NC-RTP experimentally. We use the network simulator "ns-2" [14] for all experiments and simulate. We use a simple one-hop topology, whose propagation delay is 1200 s. Such topology represents a point-to-point link between DTN Peers in interplanetary networks. Since Congestion control can not respond to the link status in time because of the long propagation delay of deep-space links, the transport protocols we use in the experiment are without congestion control, using fixed rate data transmission. In our experiment 


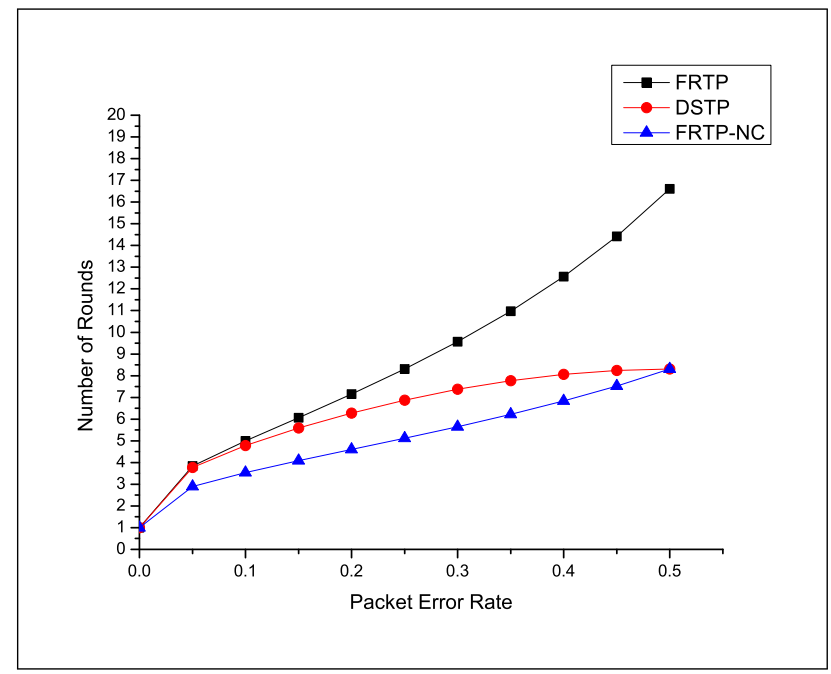

Figure 1: $R T T=2400$ s,File size $=100000$ bundles, Rounds that three protocols need in different PERs

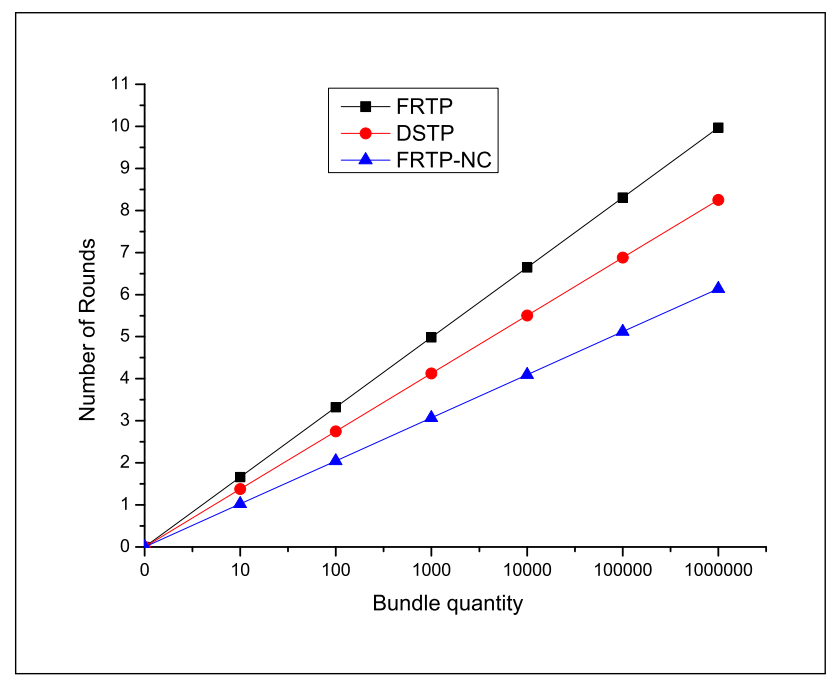

Figure 2: RTT $=2400$ s, File size $=100000$ bundles, Rounds that three protocols need in different file size

,we use FRTP, DSTP in[12], and FRTP-NC that using NC-RTP to evaluate the performance of NC-RTP. The transmit rate is 1000 bundles per second.

In Scenario 1,we evaluate the performance of NC-RTP in different PERs. The propagation delay is set to $1200 \mathrm{~s}$, file size is 100000 bundles and PER changes from 0 to 0.5 . In scenario 2 ,we evaluate the performance of NC-RTP in different file sizes. The propagation delay is still $1200 \mathrm{~s}$, PER is 0.25 and file size changes from 0 to 1000000 bundles.

From Figure 4 and Figure 5 we can get, the experimental result generally tally with the theoretical evaluation. FRTP-NC that using NC-RTP needs less time to complete file transmission than FRTP and DSTP. And with the increment of file size, the file transfer complete time of FRTP and DSTP grow sharply, FRTP-NC's transfer complete time grows slowly. So we can get a conclusion that, NC-RTP can cope with high PER in interplanetary networks and more suitable for large files, using NC-RTP can enhance transmit reliability and complete file transfers faster. 


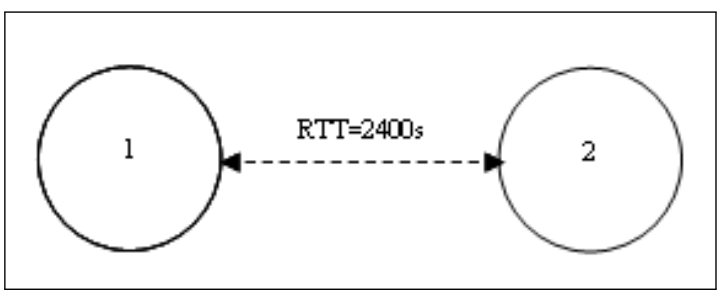

Figure 3: Topology of a point-to-point links between DTN Peers

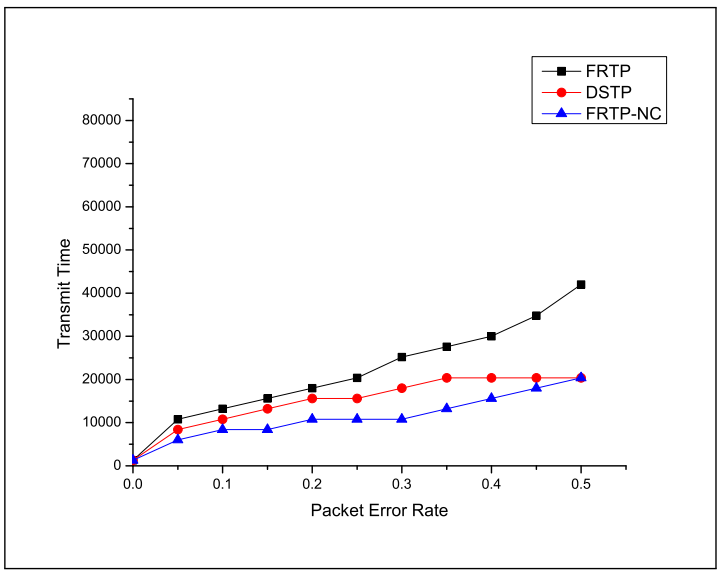

Figure 4: RTT $=2400$ s,File size $=100000$ bundles,transmit complete time change with PERs

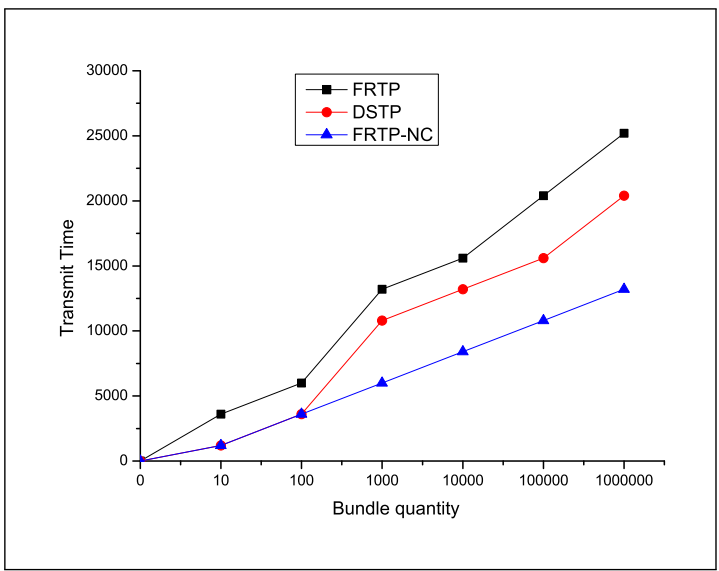

Figure 5: $\mathrm{RTT}=2400 \mathrm{~s}, \mathrm{PER}=0.25$ transmit complete time change with file size

\section{Conclusions and Future Works}

In this study, we present a Network Coding based convergence layer Reliable TransPort mechanism (NC-RTP), whose main advantage is to compensate lost bundle forwardly .NC-RTP uses fixed rate transmission and generates a redundant coded bundle periodically. The coded bundle is a random linear combination of previous bundles. Using original bundles and the coded bundle, we can decode and generate the lost original bundle with Gaussian elimination. Because of the long propagation delay of interplanetary links, using NC-RTP can enhance the transmit reliability and complete file transfers faster.

Our theoretical and simulation performance evaluation results reveal that NC-RTP presents high potential for deployability. Protocols that use NC-RTP complete file transfers faster than 
without NC-RT. we can get a conclusion that, NC-RTP can cope with high PER in interplanetary networks and more suitable for large files,using NC-RTP can enhance transmit reliability and complete file transfers faster.

Future works includes, specifically implementation on how to react to change PERs and further investigation and evaluation of the performance of NC-RTP when intermittent connectivity events happen on the transmission link.

\section{Bibliography}

[1] I.F., Akyildiz, et al.,The state of the art in interplanetary Internet Communications Magazine, IEEE Volume 42, Issue 7, July 2004 Page(s): 108118.

[2] Ian F. Akyildiz, Ozgur B. Akan, Chao Chen, Jian Fang, andWeilian Su, InterPlaNetary Internet: State-of-the-Art and Research Challenges, Computer Networks Journal (Elsevier), Vol. 43, Issue 2, pp. 75-113, October 2003.

[3] R.C. Durst, P.D. Feighery, K.L. Scott, Why not use the Standard Internet Suite for the InterPlaNetary Internet? Available from http://www.ipnsig.org/techinfo.htm.

[4] R.C. Durst, G.J. Miller, E.J. Travis, TCP extensions for space communications, Wireless Networks,Vol. 3, Issue 5, pp. 389-403, October 1997.

[5] Akan, J. Fang and I.F. Akyildiz, Performance of TCP protocols in deep space communication networks, IEEE Communications Letters, Vol. 6, Issue 11, pp.478-480, November 2002.

[6] V. Cerf, S. Burleigh et al., elay Tolerant Network Architecture,IETF RFC 4838, April 2007.

[7] K. Scott and S. Bureeigh, undle Protocol Specification IRTF DTNRG Internet Draft revision 10, July 2007

[8] O.B. Akan, J. Fang and I.F. Akyildiz, Performance of TCP protocols in deep space communication networks, IEEE Communications Letters, Vol. 6, Issue 11, pp.478-480, November 2002 .

[9] V. Tsaoussidis and I. Matta, pen issues on TCP for Mobile Computing, The Journal of Wireless Communications and Mobile Computing, WCMC John Wiley and Sons, Vol. 2, Issue 1, pp. 3-20, February 2002.

[10] L. Wood, J. McKim, W. Eddy, W. Ivancic and C. Jackson,aratoga: A Convergence Layer for Delay Tolerant Networking, IETF July 2007 meeting.

[11] M. Ramadas et al., "Licklider Transmission Protocol " Specification, IETF internet draft, April 2007

[12] Ioannis Psaras, Giorgos Papastergiou, Vassilis Tsaoussidis, and Nestor Pec-cia. DS-TP:DeepSpace Transport Protocol.IEEE Aerospace Conference, 2008, Big Sky, Montana, USA.

[13] T. Ho, "Networking from a network coding perspective,"it PhD Thesis, Massachusetts Institute of Technology, Dept. of EECS, May 2004.

[14] "ns-2 Network Simulator," http://www.isi.edu/nsnam/ns/ 